\title{
A Rigorous Study of Microstrip Crossovers and their Possible Improvements
}

\author{
Tzyy-Sheng Horng, Member, IEEE
}

\begin{abstract}
A theoretical study of microstrip crossovers is presented. For an air-bridge interconnect in a crossover configuration, the equivalent circuit examined exhibits low-pass filter characteristics. To reduce the return loss in the passband, insertion of discrete shunt capacitors is proposed and investigated. These shunt capacitors are treated as linear lumped elements and connected by via-holes of rectangular cross section. It is emphasized that air-bridges, via-holes and lumped elements used in this particular application are all rigorously included in a full-wave spectral-domain analysis.
\end{abstract}

\section{INTRODUCTION}

$\mathbf{I}$ $\mathrm{N}$ the design of microstrip circuits, crossovers are commonly used to permit two lines to cross each other with high isolation. Accurate characterization of this type of components is especially important to power and ground routing in a MMIC chip. However, up to date very few literature known to the author were devoted to modeling such components. This may be due to a complicated geometrical configuration for a single crossover consisting of a four-port junction and an air-bridge. In the recent past, the spectral-domain analysis (SDA) [1]-[9] has been developed to successfully investigate a variety of planar microstrip discontinuities up to fourport configurations. In addition, Becks and Wolff [10] have extended it to include air-bridges and via-holes of rectangular cross section, which makes SDA more promising in analyzing 3-dimensional microstrip structures.

A rigorous dispersive characterization of microstrip crossovers using SDA is performed in this paper. The analysis begins with an extraction of the equivalent circuit parameters covering a wide frequency range for a single airbridge interconnect. Upon close inspection, these parameters construct a low-pass filter prototype in which a relatively high return loss in the passband is observed. To improve the passband response, discrete shunt capacitors are inserted in the discontinuity regions, which requires a more thorough analysis in the spectral domain including an adequate treatment of linear lumped elements. In the author's previous work [11], a via-hole of rectangular cross section is used to connect a linear lumped element. The voltage across the via-hole is regarded as equivalent to the product of the lumped element impedance and the current through the via-hole. This characteristic has been skillfully put into the SDA matrix formulation such

Munuscript recelved September 30, 1993, revised April 20, 1994. This work was supported in part by the National Science Council, R.O.C., under Grant NSC82-()404-E-1 10-107

The author is with the Electrical Engineering Department, National Sun Yat-Sen University, Kaohsiung. Taiwan 80424, R.O.C.

IEEE Log Number 9404121, that the solution of the current distribution on microstrips and via-holes can reasonably account for the existence of a lumped element. Finally, the four-port scattering parameters associated with original and improved microstrip crossovers are calculated and compared to each other.

\section{SPECTRAL-DOMAIN ANALYSIS}

The spectral-domain analysis of the insertion of linear lumped elements into microstrip discontinuities is based upon the theoretical framework in [10] where the surface current densities on microstrips and the volume current density in a via hole are expanded by roof-top and uniform subdomain functions respectively. Fig. 1 shows the current-discretization procedure for a via-hole connecting a lumped element. The mathematical expressions of these current densities are given as

$$
\begin{aligned}
& j_{\mathrm{s} x}=\sum_{m} \frac{I_{x}^{m}}{\Delta y} T\left(\frac{x-x_{m}}{\Delta x}\right) P\left(\frac{y-y_{m}}{\Delta y}\right) \\
& j_{s y}=\sum_{n} \frac{I_{y}^{n}}{\Delta x} P\left(\frac{x-x_{n}}{\Delta x}\right) T\left(\frac{y-y_{n}}{\Delta y}\right) \\
& j_{z}=\frac{I_{z}}{\Delta x \Delta y} P\left(\frac{x-x_{v}}{\Delta x}\right) P\left(\frac{y-y_{v}}{\Delta y}\right) P\left(\frac{z-0.5 \Delta z}{\Delta z}\right)
\end{aligned}
$$

where

$$
T(u)= \begin{cases}1-|u|, & |u| \leq 1 \\ 0, & |u|>1\end{cases}
$$

and

$$
P(u)= \begin{cases}1, & |u| \leq \frac{1}{2} \\ 0, & |u|>\frac{1}{2}\end{cases}
$$

Note that, for $\Delta z$ much smaller than the wavelength, the use of uniform volume current distribution in a via-hole can be treated as a good approximation. Therefore, when the via-hole is used to connect a two-terminal linear lumped element, the current entering one terminal is equal to the current leaving the other terminal. This implies that Kirchhoff's current law holds. The voltage across the via-hole can be calculated as

$$
V_{z}=I_{z} Z
$$

where $Z$ is the impedance of a linear lumped element. The final step of the spectral-domain analysis results in a matrix equation:

$$
\left[\begin{array}{lll}
{\left[Z_{J_{s x} \jmath_{s x}}\right]} & {\left[Z_{j_{s x} \jmath_{s y}}\right]} & {\left[Z_{j_{s x} j_{z}}\right]} \\
{\left[Z_{J_{s y} \jmath_{s x}}\right]} & {\left[Z_{s_{s y} \jmath_{s y}}\right]} & {\left[Z_{J_{s y} j_{z}}\right]} \\
{\left[Z_{J_{z} \jmath_{s x}}\right]} & {\left[Z_{j_{z} \jmath_{s y}}\right.} & Z_{J_{z} \jmath_{z}}
\end{array}\right]\left[\begin{array}{c}
{\left[I_{x}\right]} \\
{\left[I_{y}\right]} \\
I_{z}
\end{array}\right]=\left[\begin{array}{c}
{\left[V_{x}\right]} \\
{\left[V_{y}\right]} \\
V_{z}=I_{z} Z
\end{array}\right] .
$$




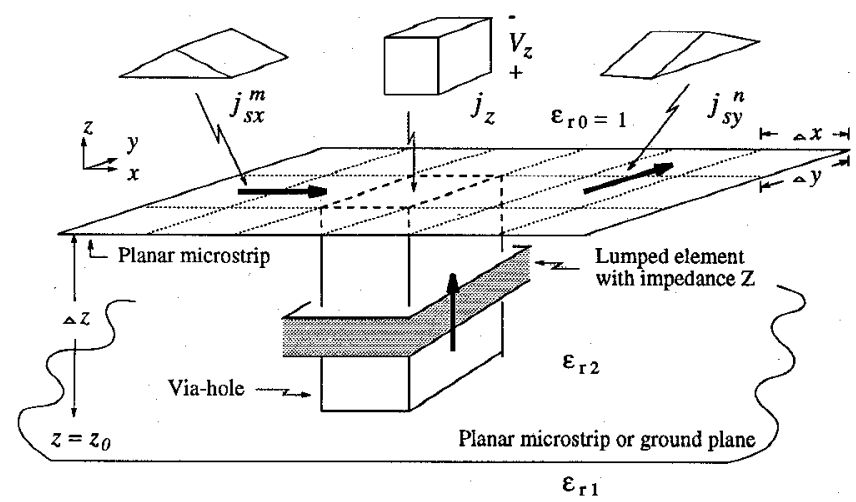

Fig. 1. Illustration of the current expansion mechanism for a via-hole connecting a linear lumped element.

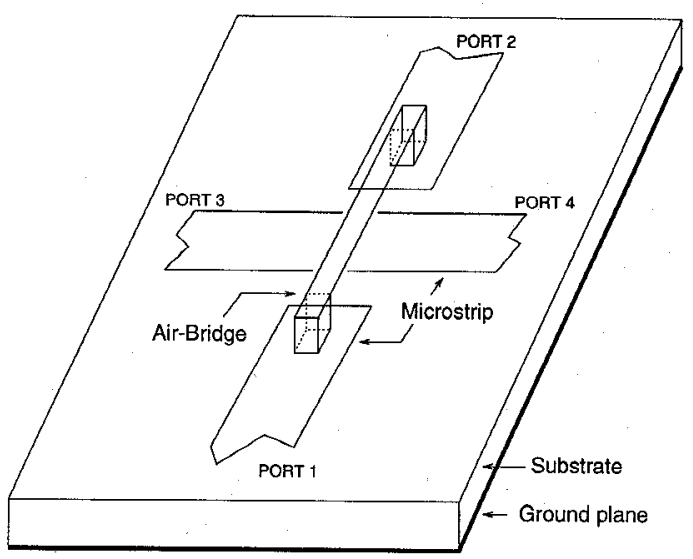

Fig. 2. Geometry of a microstrip crossover.

Each element in the left-hand side matrix of (7) is the mutual impedance between the expansion function and the testing function and is in the form of a double integration in the spectral domain. Detailed formulation of the impedance elements in the submatrices $\left[Z_{j_{s x} j_{s x}}\right],\left[Z_{j_{s x} j_{s y}}\right],\left[Z_{j_{s y} j_{s x}}\right]$ and $\left[Z_{j_{o y} j_{s y}}\right]$ can be referred to [9]. The other impedance elements containing $\hat{z}$ direct currents are evaluated efficiently using the forms given in the Appendix. On the right-hand side matrix of (7), the voltage submatrices $\left[V_{x}\right]$ and $\left[V_{y}\right]$ represent excitation vectors in the horizontal direction and contain zero-valued elements except those whose subdomain testing functions straddle a delta-gap voltage source. To solve for the coefficients of the current expansion functions, the last voltage element, $V_{z}=I_{z} Z$, needs to be moved into the left-hand side matrix and leaves a zero value instead. Correspondingly, the value of the impedance element $Z_{j_{z} j_{z}}$ is modified as

$$
Z_{j_{z} j_{z}}^{\prime}=Z_{j_{z} j_{z}}-Z
$$

\section{AIR-BRIDGE INTERCONNECTS}

Fig. 2 shows the configuration of a microstrip crossover on a dielectric substrate backed by a ground metallization. To avoid unwanted metallic contact with port 3 and port 4, port 1 and port 2 are connected with an air-bridge. Empirically, the air-bridge shows obvious discontinuity effects at high frequencies, which may obstruct transmission of signals from
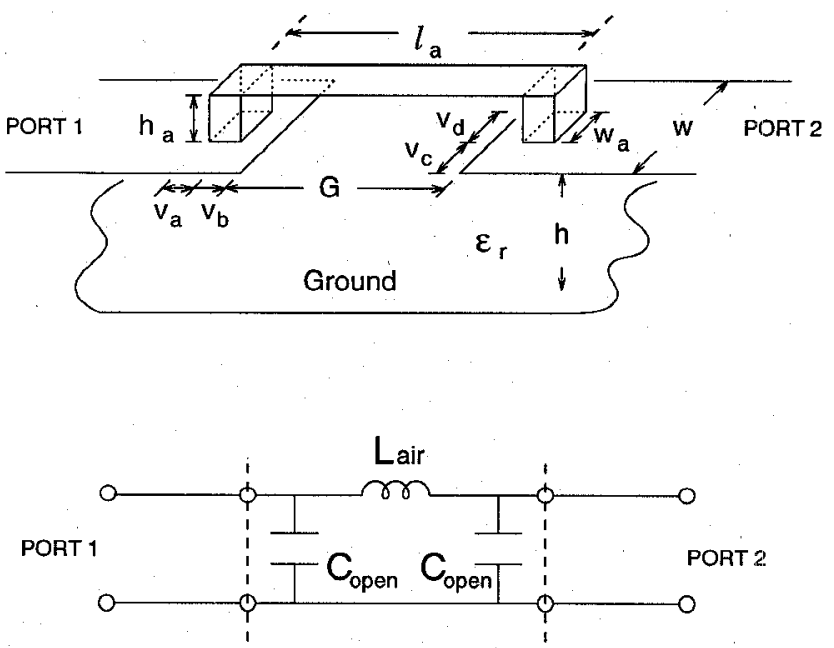

Fig. 3. Schematic view on an air-bridge interconnect. Geometrical parameters: $\epsilon_{r}=9.8, w=635 \mu \mathrm{m}, h=635 \mu \mathrm{m}, G=1905 \mu \mathrm{m}, l_{a}=2540 \mu \mathrm{m}$, $h_{a}=200 \mu \mathrm{m}, w_{a}=211.67 \mu \mathrm{m}, v_{a}=v_{b}=v_{c}=v_{d}=211.67 \mu \mathrm{m}$.

port 1 to port 2 and vice versa. Therefore, a full-wave study of such interconnects for better understanding and possible improvements is highly demanded. As an example, Fig: 3 shows an air-bridge printed on a $635 \mu \mathrm{m}$ ceramic substrate with a conductor width of $635 \mu \mathrm{m}$ at both port 1 and port 2 . The corresponding characteristic impedance of such lines is approximately $50 \Omega$. The gap width is three times the substrate thickness and the bridge height and length are $200 \mu \mathrm{m}$ and $2540 \mu \mathrm{m}$ respectively. With a choice of reference planes right at the centers of the vertically directed parts of the air-bridge, the equivalent $\pi$ network parameters are evaluated and plotted in Fig. 4. It is noted that, in the frequency range of interest, the losses launched from the discontinuity are negligible. Thus only reactive elements are included in the equivalent circuit. Intuitively, the equivalent circuit for an air-bridge interconnect can be regarded as an air-bridge inductance $L_{\text {air }}$ in conjunction with two open-end capacitances $C_{\text {open }}$, which constructs a three-element low-pass filter in $\pi$ form. From Fig. 4 one can see that the values of $L_{\text {air }}$ vary from $1.69 \mathrm{nH}$ to $1.17 \mathrm{nH}$ and the values of $C_{\text {open }}$ vary from $0.104 \mathrm{pF}$ to $0.119 \mathrm{pF}$ as the frequency increases from $2 \mathrm{GHz}$ to $20 \mathrm{GHz}$. From filter theory [12], two parameters are commonly used to estimate a filter response. One is the cutoff frequency $f_{c}$ which is the upper bound of the passband frequency range. The other is the image impedance $Z_{i}$ which is defined by the terminating impedance for port 2 with an input match at port 1, or vice versa. Mathematical expressions of both parameters are given as

$$
\begin{aligned}
& f_{c}=\frac{1}{\pi \sqrt{L C}}, \\
& Z_{i}=\frac{\sqrt{\frac{L}{C}}}{\sqrt{1-\frac{f^{2}}{f_{c}^{2}}}} .
\end{aligned}
$$

For $f<f_{c}$, equation (10) shows that $Z_{i}$ is pure real. This implies that port 1 and port 2 can be matched in the passband if the characteristic impedance of the microstrip lines at both ports is equal to the real $Z_{i}$. As for $f>f_{c}$, the values of 
TABLE I

Cut-OfF Frequencies and Image IMPEDANCEs of THE STRUcture SHOwn IN Fig. 3

\begin{tabular}{|c|c|c|c|c|c|}
\hline$f(\mathrm{GHz})$ & 2 & 4 & 6 & 8 & 10 \\
\hline$f_{c}(\mathrm{GHz})$ & 23.98 & 23.99 & 24.01 & 24.03 & 24.25 \\
\hline$Z_{\imath}(\Omega)$ & 12793 & 129.25 & 131.21 & 133.12 & 135.06 \\
\hline \hline$f(\mathrm{GHz})$ & 12 & 14 & 16 & 18 & 20 \\
\hline$f_{c}(\mathrm{GHz})$ & 2457 & 24.97 & 25.46 & 26.18 & 26.99 \\
\hline$Z_{\imath}(\Omega)$ & 136.38 & 138.89 & 142.82 & 145.10 & 14715 \\
\hline
\end{tabular}

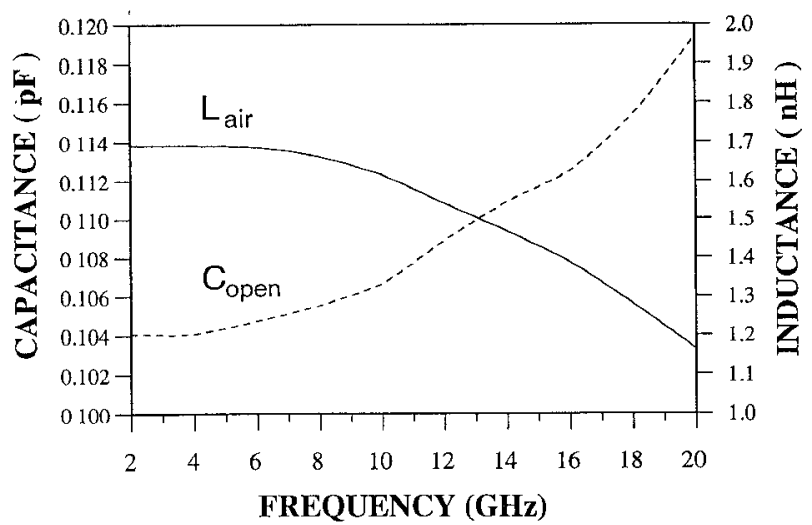

Fig. 4. Equivalent circuit parameters of the structure shown in Fig. 3.

$Z_{\imath}$ become pure imaginary, which leads to an attenuation rate for the transmitted power. Thus this frequency region corresponds to the stopband of the filter section. Substituting the element values provided in Fig. 4 into (9) and (10), both filter parameters associated with the structure in Fig. 3 are obtained and shown in Table I. It is found that all frequencies of interest are below cutoff; however, the image impedances listed are much larger than $50 \Omega$, which results in a significant mismatch in the passband.

From (10) an increase of capacitance can effectively lower the image impedances. Practically, this can be implemented in two different ways. One way is to insert a pair of shunt capacitors with capacitance $C_{\mathrm{sh}}$ each into the regions near the open-ends (see Fig. 5). The other way is to enlarge each open-end area, for example, as a $S \times S$ square (see Fig. 6 ). As a result, the magnitudes of the scattering parameters for both structures are calculated with the SDA method and plotted in Fig. 7 and Fig. 8 respectively. It can be seen that, when compared to the results of the cases without any changes $\left(C_{\mathrm{sh}}=0 \mathrm{pF}\right.$ or $\left.S=W\right)$, the return loss is dramatically reduced at low frequencies as $C_{\mathrm{sh}}$ or $S$ is increased. Nevertheless, these improvements are at the expense of a narrower passband. To remedy this drawback, another structure corresponding to a higher-order low-pass filter prototype is suggested in Fig. 9, where the geometry shown is the same as that in Fig. 5 except for a shift of individual via-hole containing a capacitor toward the center of the gap. The corresponding SDA results are shown in Fig. 10. Comparing with the results in Fig. 7, one observes that this new structure not only enhances the passband width but also alleviates the return loss.
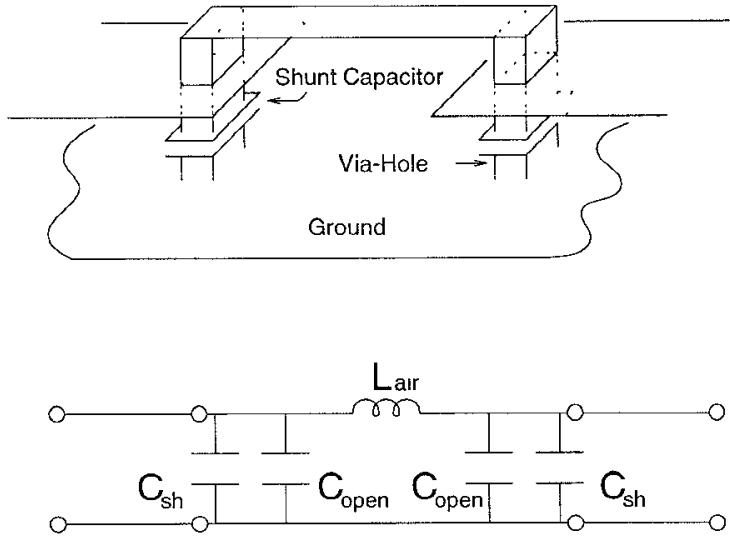

Fig. 5. Schematic view on an air-bridge interconnect with shunt capacitors. Geometrical parameters are provided in Fig. 3.
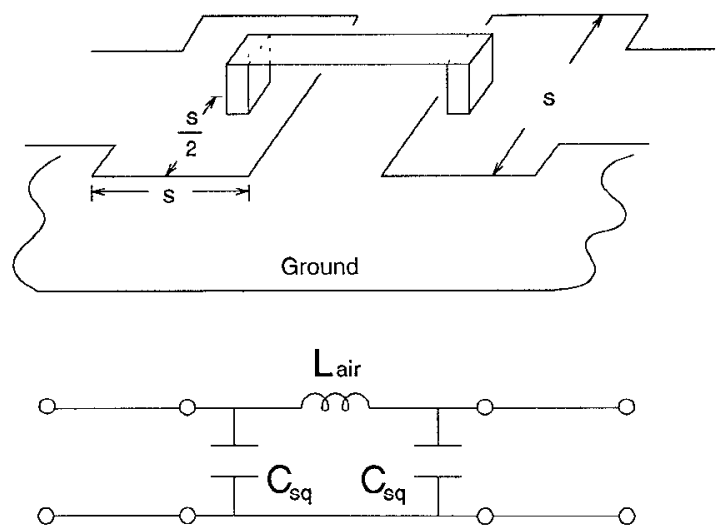

Fig. 6. Schematic view on an air-bridge interconnect with enlarged open-end areas. Geometrical parameters are provided in Fig. 3.

\section{MiCROSTRIP CROSSOVERS}

Fig. 11 shows the top vicws of two crossover structures whose air-bridge interconnects are shown in Fig. 3 and Fig. 9 respectively. From the results demonstrated in section III, the former crossover is clearly inferior to the latter if the return loss measured at port 1 is the criterion. However, in this section one more factor, the isolation of port 1 from port 3/port 4 , is included for more rigorous judgement. In general, solution of the scattering parameters for a microstrip junction needs knowledge of the surface current density on the junction resulting from suitable excitation and impedance matching. Therefore, in the analysis port 1 is assumed to be excited by a voltage generator, whereas the rest of ports are assumed to be terminated by matched loads with complex impedances. Note that a full-wave technique is employed to model such generators and loads by referring to [11]. Under this scheme, the calculated current distributions along the microstrip lines of four ports can be used to extract $S_{p 1}$ for $p=1, \ldots, 4$. As a result, Fig. 12 shows the magnitude of these scattering parameters. It is found that the structure containing the capacitors has a larger magnitude of $S_{31}$ (or $S_{41}$ ) when frequency is below $8 \mathrm{GHz}$ or above $16 \mathrm{GHz}$.

\section{CONClusion}

A rigorous full-wave study of microstrip crossovers and their possible improvements has been presented. The method 


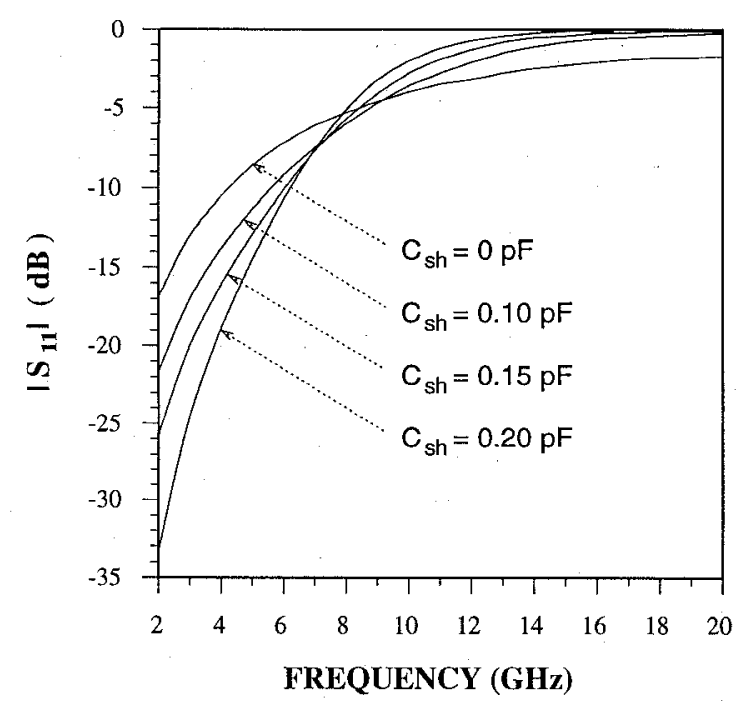

Fig. 7. Computed return loss of the structure shown in Fig. 5.

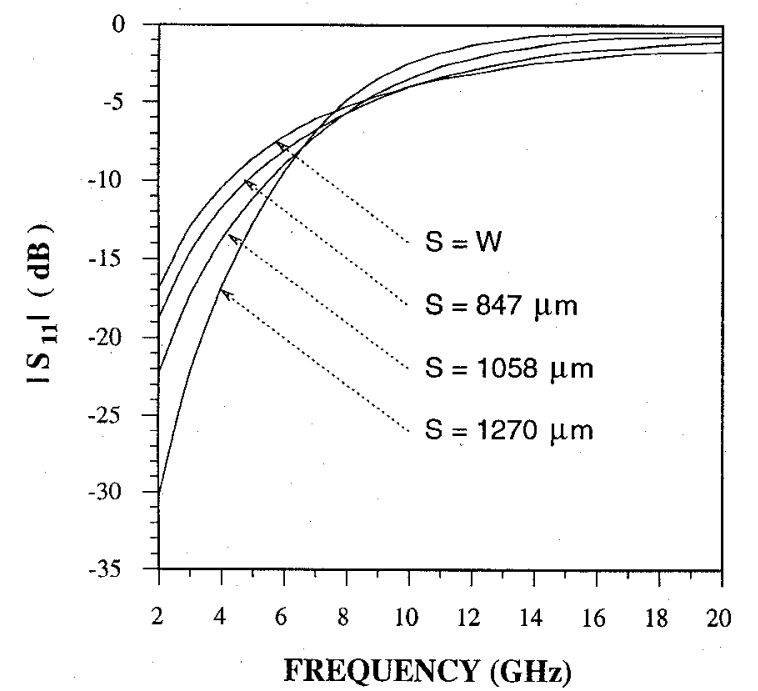

Fig. 8. Computed return loss of the structure shown in Fig. 6.
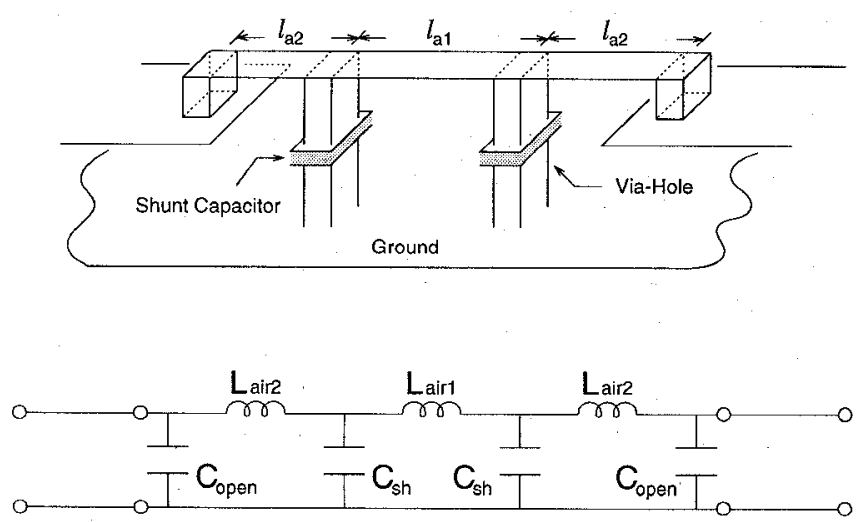

Fig. 9. Schematic view on an air-bridge interconnect corresponding to a seven-element low-pass filter. Geometrical parameters are provided in Fig. 3 except $l_{a 1}=1270 \mu \mathrm{m}$ and $l_{a 2}=635 \mu \mathrm{m}$

used is based on an advanced spectral-domain technique which can accurately characterize vias and hybrid linear elements

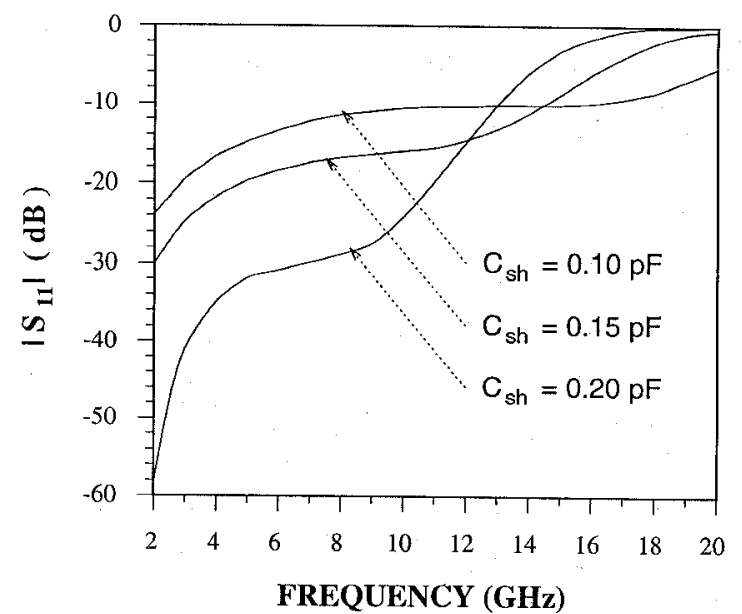

Fig. 10. Computed return loss of the structure shown in Fig. 9.
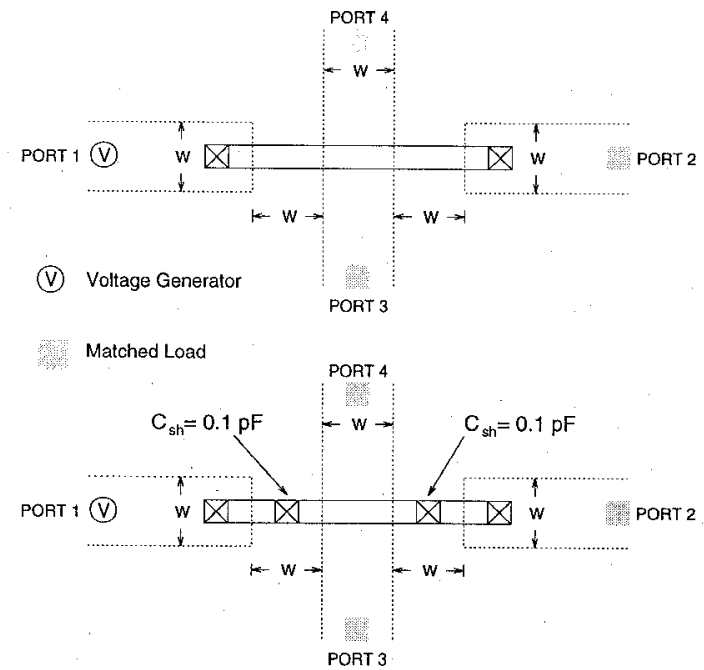

Fig. 11. Top: Top view of a microstrip crossover. Bottom: Top view of a capacitor-loaded microstrip crossover. Geometrical parameters of the air-bridges are provided in Figs. 3 and 9.

in a microstrip structure. Numerical results demonstrate that, by properly inserting shunt capacitors or enlarging the openend areas, the mismatch for an air-bridge interconnect in a crossover can be alleviated substantially.

\section{APPENDIX}

The impedance elements in $\left[Z_{j_{s x} j_{z}}\right],\left[Z_{j_{s y} j_{z}}\right],\left[Z_{j_{z} j_{s x}}\right]$ and $\left[Z_{j_{z} j_{s y}}\right]$ are given as follows:

$$
\begin{aligned}
Z_{j_{s x}^{m} j_{z}}= & Z_{j_{z} j_{s x}^{m}}=\frac{1}{\pi^{2} \Delta x(\Delta y)^{2}} \int_{0}^{\infty} \int_{0}^{\infty} \tilde{G}_{x z}\left(k_{x}, k_{y}\right) \\
& \times \tilde{T}\left(k_{x}\right) \tilde{P}\left(k_{x}\right) \tilde{P}^{2}\left(k_{y}\right) \sin k_{x}\left(x_{v}-x_{m}\right) \\
& \times \cos k_{y}\left(y_{v}-y_{m}\right) d k_{x} d k_{y} \\
Z_{j_{s y}^{n} j_{z}}= & Z_{j_{z} j_{s y}^{n}}=\frac{1}{\pi^{2}(\Delta x)^{2} \Delta y} \int_{0}^{\infty} \int_{0}^{\infty} \tilde{G}_{x z}\left(k_{y}, k_{x}\right) \\
& \times \tilde{T}\left(k_{y}\right) \tilde{P}\left(k_{y}\right) \tilde{P}^{2}\left(k_{x}\right) \cos k_{x}\left(x_{v}-x_{n}\right) \\
& \times \sin k_{y}\left(y_{v}-y_{n}\right) d k_{x} d k_{y}
\end{aligned}
$$




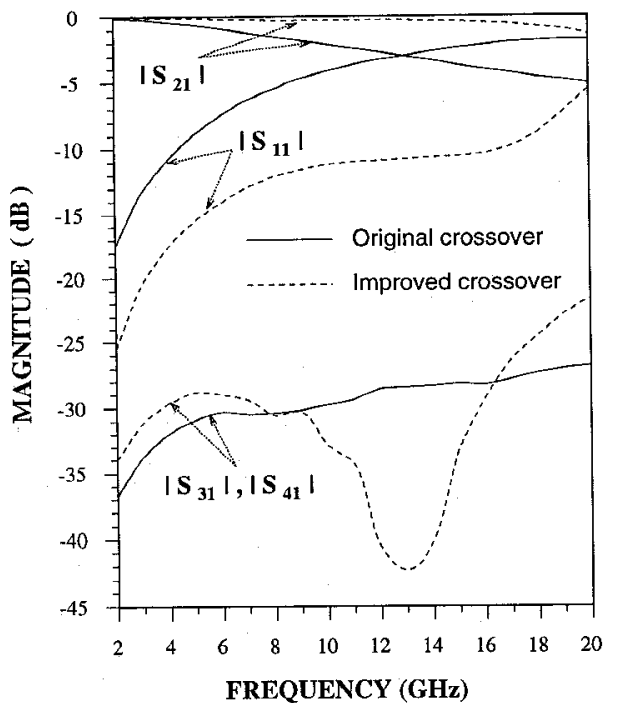

Fig. 12. Magnitude of the scattering parameters of the structures shown in Fig. 11.

where

$$
\begin{aligned}
\tilde{G}_{x z}\left(k_{x}, k_{y}\right)= & \frac{k_{x} Z_{0}}{k_{0} T_{m}\left(k_{x}, k_{y}\right)} \operatorname{Sgn}\left[D\left(k_{x}, k_{y}\right) \sinh q_{2} \Delta z\right. \\
& \left.+\frac{q_{0} q_{1}}{q_{2}^{2}} \sinh q_{1} z_{0}\left(-1+\cosh q_{2} \Delta z\right)\right],(\mathrm{A}
\end{aligned}
$$

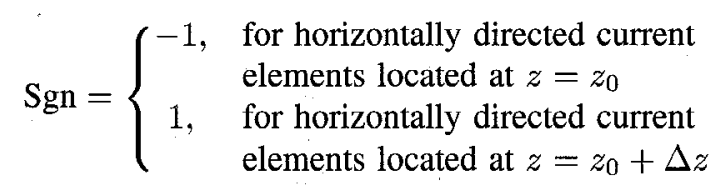

$$
D\left(k_{x}, k_{y}\right)= \begin{cases}\frac{q_{1}}{\epsilon_{r 2} q_{2}} \sinh q_{1} z_{0}, & \text { for horizontally directed } \\ & \text { current elements located } \\ & \text { at } z=z_{0} \\ \frac{\epsilon_{r 1} q_{0}}{\epsilon_{r 2} q_{2}} \cosh q_{1} z_{0}, & \text { for horizontally directed } \\ & \text { current elements located } \\ & \text { at } z=z_{0}+\Delta z\end{cases}
$$

$$
\begin{aligned}
T_{m}\left(k_{x}, k_{y}\right)= & q_{1} \sinh q_{1} z_{0} \cosh q_{2} \Delta z \\
& +\frac{\epsilon_{r 2} q_{0} q_{1}}{q_{2}} \sinh q_{1} z_{0} \sinh q_{2} \Delta z \\
& +\frac{\epsilon_{r 1} q_{2}}{\epsilon_{r 2}} \cosh q_{1} z_{0} \sinh q_{2} \Delta z \\
& +\epsilon_{r 1} q_{0} \cosh q_{1} z_{0} \cosh q_{2} \Delta z
\end{aligned}
$$

and

$$
q_{i}=\sqrt{k_{x}^{2}+k_{y}^{2}-k_{0}^{2} \epsilon_{r i}}, \quad i=0,1,2
$$

The impedance element $Z_{j_{z} j_{z}}$ is expressed as

$$
\begin{aligned}
Z_{j_{z} j_{z}}=\frac{1}{\pi^{2}(\Delta x \Delta y)^{2}} \int_{0}^{\infty} \int_{0}^{\infty} \tilde{G}_{z z}\left(k_{x}, k_{y}\right) \tilde{P}^{2}\left(k_{x}\right) \\
\times \tilde{P}^{2}\left(k_{y}\right) d k_{x} d k_{y}
\end{aligned}
$$

where

$$
\begin{aligned}
\tilde{G}_{z z}\left(k_{x}, k_{y}\right)= & \frac{-j Z_{0} k_{0} \Delta z}{q_{2}^{2}} \\
& +\frac{j Z_{0}\left(k_{x}^{2}+k_{y}^{2}\right)}{k_{0} \epsilon_{r 2} T_{m}\left(k_{x}, k_{y}\right)}\left[\frac{q_{1}}{q_{2}^{3}} \sinh q_{1} z_{0} \sinh q_{2} \Delta z\right. \\
& +\frac{2 \epsilon_{r 2} q_{0} q_{1}}{q_{2}^{4}} \sinh q_{1} z_{0}\left(-1+\cosh q_{2} \Delta z\right) \\
& \left.+\frac{\epsilon_{r 1} q_{0}}{q_{2}^{3}} \cosh q_{1} z_{0} \sinh q_{2} \Delta z\right]
\end{aligned}
$$

Set $z_{0}=0$ and $\epsilon_{r 1}=\epsilon_{r 2}$ in the above equations if the ground plane is placed at $z=z_{0}$.

\section{ACKNOWLEDGMENT}

The author would like to thank Dr. Chi-Yang Chang for many helpful insights and technical discussions.

\section{REFERENCES}

[1] R. H. Jansen, "The spectral-domain approach for microwave integrated circuits," IEEE Trans. Microwave Theory Tech., vol. MTT-33, pp. 1043-1056, Oct. 1985.

[2] R. W. Jackson and D. M. Pozar, "Full wave analysis of microstrip openend and gap discontinuities," IEEE Trans. Microwave Theory Tech., vol. MTT-33, pp. 1036-1042, Oct. 1985.

[3] H. Y. Yang and N. G. Alexopoulos, "Basic blocks for high frequency interconnects: Theory and experiment," IEEE Trans. Microwave Theory Tech., vol. MTT-36, pp. 1258-1264, Aug. 1988.

[4] H. Y. Yang, N. G. Alexopoulos, and D. R. Jackson, "Microstrip openend and gap discontinuities in a substrate-superstrate structure," IEEE Trans. Microwave Theory Tech., vol. 37, pp. 1542-1546, Oct. 1989.

[5] R. W. Jackson, "Full-wave, finite element analysis of irregular microstrip discontinuities," IEEE Trans. Microwave Theory Tech., vol. 37, pp. 81-89, Jan. 1989.

[6] S. C. Wu, H. Y. Yang, N. G. Alexopoulos, and I. Wolff, "A rigorous dispersive characterization of microstrip cross and T junctions," IEEE Trans. Microwave Theory Tech., vol. 38, pp. 1837-1844, Dec. 1990.

[7] T. S. Horng, W. E. McKinzie, and N. G. Alexopoulos, "Full-wave spectral-domain analysis of compensation of microstrip discontinuities using triangular subdomain functions," IEEE Trans. Microwave Theory Tech., vol. 40, pp. 21377-2147, Dec. 1992.

[8] T. S. Horng, N. G. Alexopoulos, S. C. Wu and H. Y. Yang, "Full-wave spectral-domain analysis for open microstrip discontinuities of arbitrary shape including radiation and surface-wave losses," Int. J. Microwave Millimeter-wave Computer-Aided Eng., vol . 2, no. 4. pp. 224-240, 1992.

[9] S. C. Wu, "Full-wave spectral-domain analysis of microstrip antennas and circuit discontinuities," Ph.D. Dissertation, University of California, Los Angeles, 1991.

[10] T. Becks and I. Wolff, "Analysis of 3-D metallization structures by a full-wave spectral-domain technique," IEEE Trans. Microwave Theory Tech., vol. 40, pp. 2219-2227, Dec. 1992.

[11] T. S. Horng, "Extending the three-dimensional spectral-domain approach to hybrid microwave integrated circuits with passive and active lumped elements," accepted for publication in 1994 IEEE MTT-S Int. Microwave Symp. Dig., pp. 709-712, 1994.

[12] D. M. Pozar, Microwave Engineering. 'Reading, MA: Addison-Wesley, 1990

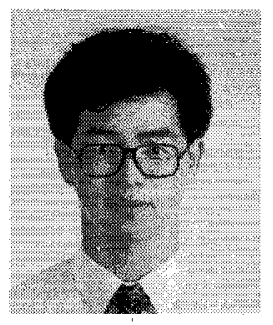

Tzyy-Sheng Horng (S'88-M'92) was born December 7, 1963, in Taichung, Taiwan. He received the B.S.E.E. degree from the National Taiwan University, and the M.S.E.E. and Ph.D. degrees from the University of California, Los Angeles, in 1985, 1990, and 1992, respectively.

Since 1992, he has been an Associate Professor of the Electrical Engineering Department at National Sun Yat-Sen University, Kaohsiung, Taiwan. His research interests is in the area of electromagnetic simulations of microwave integrated cirucits. 